\title{
Epidemiology of oligometastatic non-small cell lung cancer: results from a systematic review and pooled analysis
}

\author{
Elisa Gobbini ${ }^{1,2}$, Luca Bertolaccini ${ }^{3}$, Niccolò Giaj-Levra ${ }^{4}$, Jessica Menis ${ }^{5}$, Matteo Giaj-Levra ${ }^{2,6} \wedge$ \\ ${ }^{1}$ Cancer Research Center Lyon, Center Léon Bérard, Lyon, France; ${ }^{2}$ Thoracic Oncology Unit, Grenoble University Hospital, Grenoble, France; \\ ${ }^{3}$ Division of Thoracic Surgery, IEO, European Institute of Oncology IRCCS, Milan, Italy; ${ }^{4}$ Department of Advanced Radiation Oncology, IRCCS \\ Sacro Cuore Don Calabria Hospital, Negrar di Valpolicella, Italy; ${ }^{5}$ Section of Oncology, Department of Medicine, University of Verona Hospital \\ Trust, Verona, Italy; 'Institute for Advanced Biosciences INSERM U1209 CNRS UMR5309, Université Grenoble Alpes, France \\ Contributions: (I) Conception and design: All authors; (II) Administrative support: All authors; (III) Provision of study materials or patients: E \\ Gobbini, L Bertolaccini, N Giaj-Levra, M Giaj-Levra; (IV) Collection and assembly of data: E Gobbini, L Bertolaccini, N Giaj-Levra, M Giaj- \\ Levra; (V) Data analysis and interpretation: All authors; (VI) Manuscript writing: All authors; (VII) Final approval of manuscript: All authors. \\ Correspondence to: Matteo Giaj-Levra, MD, PhD. Thoracic Oncology Unit, Grenoble University Hospital, CS 10217, 38043 Grenoble, France. \\ Email: matteo.giajlevra@orange.fr.
}

Background: To describe the incidence and the clinical characteristics of oligometastatic non-small cell lung
cancer (NSCLC) patients. Oligometastatic NSCLC is gaining recognition as a clinical condition with a different
prognosis compared to multi metastatic disease. Usually, four different scenarios of oligometastatic disease can be
described but not epidemiological data are available. To date, it is difficult to delineate an exhaustive epidemiological
scenario because no uniform or shared definition of oligometastatic status exists, even though a recent consensus
defined synchronous oligometastatic disease as having a maximum of 5 metastases in 3 different organs. Methods: A systematic review and a pooled analysis of literature were performed. Article selection was based on the following characteristics: focus on lung cancers; dealing with oligometastatic settings and providing a definition of oligometastatic disease; number of metastatic lesions with or without the number of organs involved; providing some incidence or clinical characteristics of oligometastatic NSCLC patients. Series focusing on a specific single metastatic organ were excluded. The research was launched in MEDLINE (OvidSP) in March 2020. Full articles were individually and collectively read by the authors according to the previous criteria. Each author inspected the reference list included in the eligible articles. If the selection criteria were recognized, the article was reviewed by all authors and then included. Data on patient clinical features were pooled together from 31 articles selected.

Results: A total number of 31 articles have been selected for the analysis. The following variables were extracted from the publications: (I) number of metastases, (II) number of organs involved, (III) number of patients, (IV) number and percentage of males and females, (V) number and percentage of squamous and non-squamous histology, (VI) $\mathrm{T}$ and $\mathrm{N}$ status and/or stage of primary disease for oligometastatic setting. The data collected have been analyzed according to the oligometastatic setting.

Conclusions: Oligometastatic status is globally identified as a different clinical condition from multi metastatic NSCLC, although the clinical characteristics were consistent in the general metastatic population, even with a lower-than-expected TN status. The brain and bones were the most frequent organs involved. Lacking consensus definition, these results must be interpreted cautiously and a prospective evaluation is urgently needed.

Keywords: Non-small cell lung cancer (NSCLC); oligometastatic disease; epidemiology; incidence

Submitted Aug 31, 2020. Accepted for publication Jul 07, 2021.

doi: $10.21037 /$ tlcr-20-982

View this article at: https://dx.doi.org/10.21037/tlcr-20-982

$\wedge$ ORCID: 0000-0002-9015-6871. 


\section{Introduction}

Oligometastatic status is gaining recognition as a narrow clinical disease condition that requires an efficient, multidisciplinary management approach. In the oligometastatic setting, four different scenarios can be described overall: synchronous, oligo-persistent, oligoprogressive, and oligo-recurrent disease, depending whether the oligometastatic disease is diagnosed during a treatmentfree interval or active systemic therapy $(1,2)$. Each of these disease conditions should be handled differently, with the choice of the most appropriate therapeutic sequence being the major challenge. Systemic therapies with local ablative treatments can potentially eradicate a limited number of metastatic foci or prevent a therapeutic switch, theoretically postponing disease progression (3). In fact, Ashworth et al (4) found favorable survival by adding local surgery or radical dose of radiotherapy (RT) to metastatic lesions.

The $8^{\text {th }}$ edition of the non-small cell lung cancer (NSCLC) TNM classification first discussed the existence of synchronous oligo-metastatic patients with a single extrathoracic metastatic lesion (M1b), quite similar in prognosis to those presenting intra-thoracic metastatic disease only (M1a) (5). This group clearly stood out from the rest of the metastatic population (M1c), presenting multiple metastatic organs with single or multiple lesions and a worse prognosis. The identification of this new patient category can be linked to several factors, including improvement in diagnostic and imaging accuracy, the continuous increase in systemic oncological treatment efficacy, and the favorable tolerability of local ablative treatments (6).

In literature data, each published case series and clinical trial has employed its own definition of oligometastatic disease without comparable inclusion criteria. More often than not, a particular metastatic localization or a specific local treatment are selected, making an exhaustive picture impossible.

Recently, a consensus in the European Organisation for Research and Treatment of Cancer (EORTC) and the European Society of Radiotherapy (ESTRO) defined synchronous oligometastatic disease as having a maximum of 5 metastases in 3 different organs $(1,2)$. This certainly helps in making future clinical trials more comparable. The present article first reviews literature on the incidence, demographics and distribution of oligometastatic patients. Then, a pooled analysis of clinical data from selected case series attempts to better describe this population. We present the following article in accordance with the PRISMA reporting checklist (available at https://dx.doi. org/10.21037/tlcr-20-982).

\section{Materials and methodology for the systematic review and pooled analysis}

The key focus of this literature review is the incidence and clinical characteristics of oligometastatic NSCLC patients. The standard reporting guidelines [Preferred Reporting Items for Systematic Reviews and Meta-Analyses statement (http://www.prisma-statement.org/)] are used. The research was launched in MEDLINE (OvidSP) in March 2020. January 1, 1996 was established as the cutoff time for articles. All titles and abstracts were screened individually and then collectively reviewed. Only contributions in English were accepted, and disagreements were resolved by consensus.

The authors considered abstracts eligible if: (I) focused on lung cancers, (II) dealt with oligometastatic settings and provided a definition of oligometastatic disease [synchronous oligometastatic disease (SOM), oligorecurrent (ORD), oligoprogressive diseases (OPD), and oligo-persistent disease $(\mathrm{OPeD})$, number of metastatic lesions with or without the number of organs involved], (III) provided some incidence or clinical characteristics of oligometastatic NSCLC patients. Series focusing on a specific single metastatic organ were excluded.

Then, full articles were individually and collectively read by the authors according to the previous criteria. Each author inspected the reference list included in the eligible articles. If the selection criteria were recognized, the article was reviewed by all authors and then included.

The following variables were extracted from the publications: (I) number of metastases, (II) number of organs involved, (III) number of patients, (IV) number and percentage of males and females, (V) number and percentage of squamous and non-squamous histology, (VI) and $\mathrm{N}$ status and/or stage of primary disease for SOM and ORD, and of initial disease at diagnosis for OPD and OpeD. Data concerning patient clinical features were pooled together. The results are expressed as median and range for quantitative variables, with frequencies or percentages $[\mathrm{n}(\%)]$ for qualitative variables. All analyses were carried out using XLStat software (2018 Version).

\section{Clinical characteristics of oligometastatic patients according to literature data and the pooled analysis}

A systematic review published by Ashworth et al. explored the outcomes and prognostic factors in 2,176 synchronous and/or metachronous oligometastatic NSCLC patients 


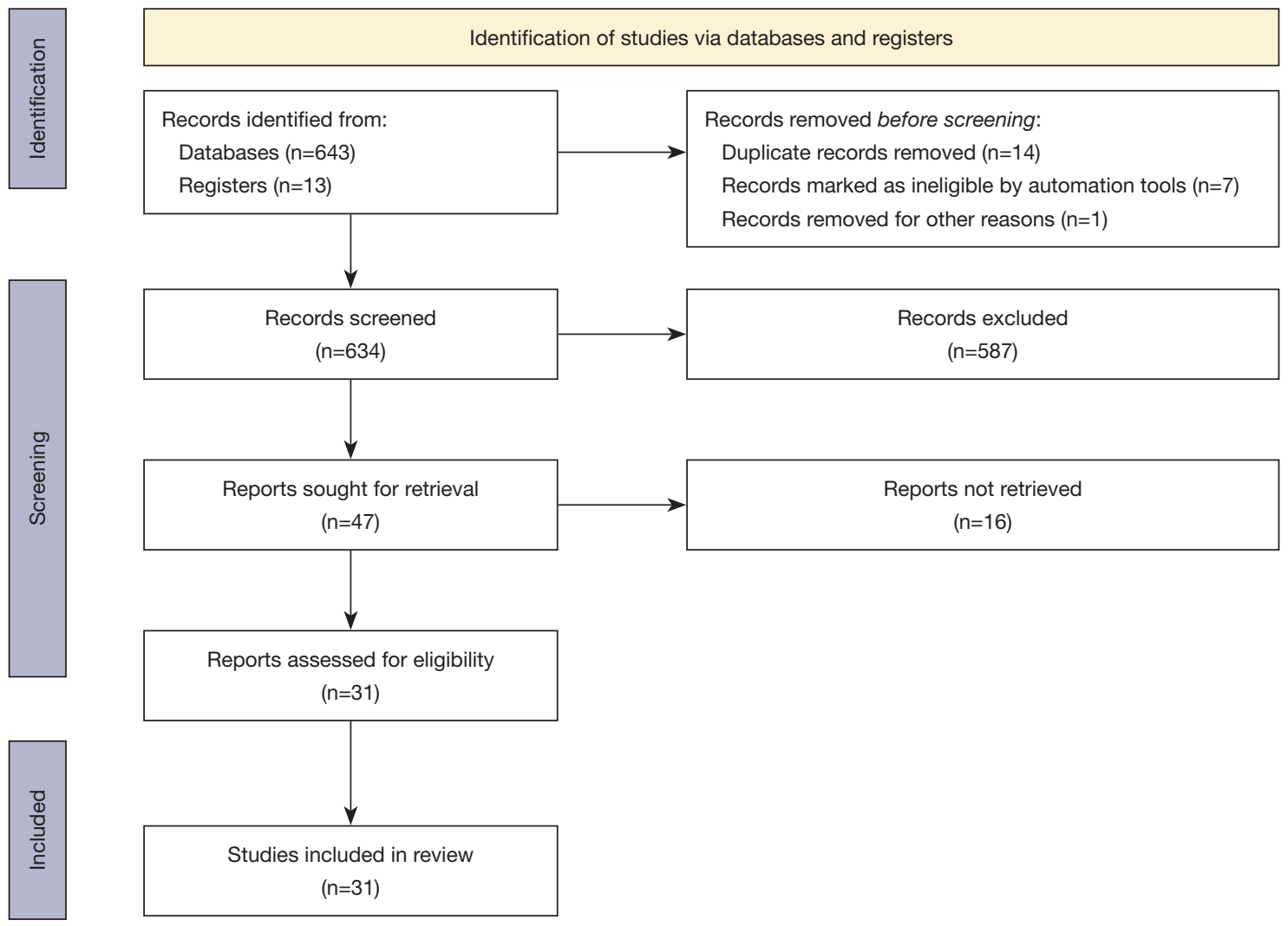

Figure 1 PRISMA 2020 flow diagram for new systematic reviews which included database and register searches only.

with up to 5 metastatic lesions, providing interesting data on the patients' clinical characteristics (7). Notably, the patients were younger compared to the general NSCLC population. In fact, approximately half of the studies in the Ashworth et al. review reported a median age of 52 to 69 years, with the rest reporting a mean age of 52 to 67.8 years. Adenocarcinoma was the most represented histology $(58.8 \%)$, and more than half of the patients had a solitary metastasis, with $85 \%$ presenting 3 or fewer metastases. A significant proportion of patients (35.7\%) had brain metastases. The Karnofsky (KPS) or ECOG performance status (ECOG PS) was reported in only half of the studies, and most of them included patients with a KPS $\geq 70$ or an ECOG PS of 1 or better only. Consequently, no information about the general clinical condition of these patients can be determined. Given $60 \%$ of the review's studies focused on patients with a brain metastasis only, and just $16.3 \%$ of its studies included patients with up to 5 metastases, the review is quite limited. Articles that included patients with a variety of metastatic organs involved represented almost one-quarter of the total.
In our systematic review, we excluded series focusing on a single organ and separately analysed the different oligometastatic disease conditions to provide a more exhaustive portrait. We selected 31 articles (Figure 1), including 2,208 oligometastatic NSCLC patients. Twentyone articles focused on SOM $(\mathrm{N}=1,514)(8-28)$, four on OPD $(\mathrm{N}=320)$ (29-32), four on ORD $(\mathrm{N}=296)(33-36)$ and two on OpeD $(\mathrm{N}=78)(37,38)$ (Tables 1-4). The patients' clinical features here resembled the expected features of the metastatic NSCLC general population. The patients had a median age of 61 years (range, 55-71 years), $52 \%$ $(\mathrm{N}=1,136)$ were men, and $84 \%(\mathrm{~N}=1,751)$ had a nonsquamous histology. Even then, individual TNM staging was lacking in most cases, and primary disease seemed more limited than usual. Indeed, TNM staging was classified as $\mathrm{N} 0-\mathrm{N} 1$ and $\mathrm{N} 2-\mathrm{N} 3$ in $41 \%(\mathrm{~N}=591)$ and $57 \%(\mathrm{~N}=822)$ of cases respectively, and as $\mathrm{T} 0-\mathrm{T} 1$ in $55 \%(\mathrm{~N}=766)$ and T2-T4 $(\mathrm{N}=593)$ in $43 \%$ of patients. Patients most often had brain $(48 \%)$ or bone $(26 \%)$ metastases, whereas the adrenal gland and liver were involved in $12 \%$ and $5 \%$ of cases, respectively (Figure 2). Metastases to the lung were 


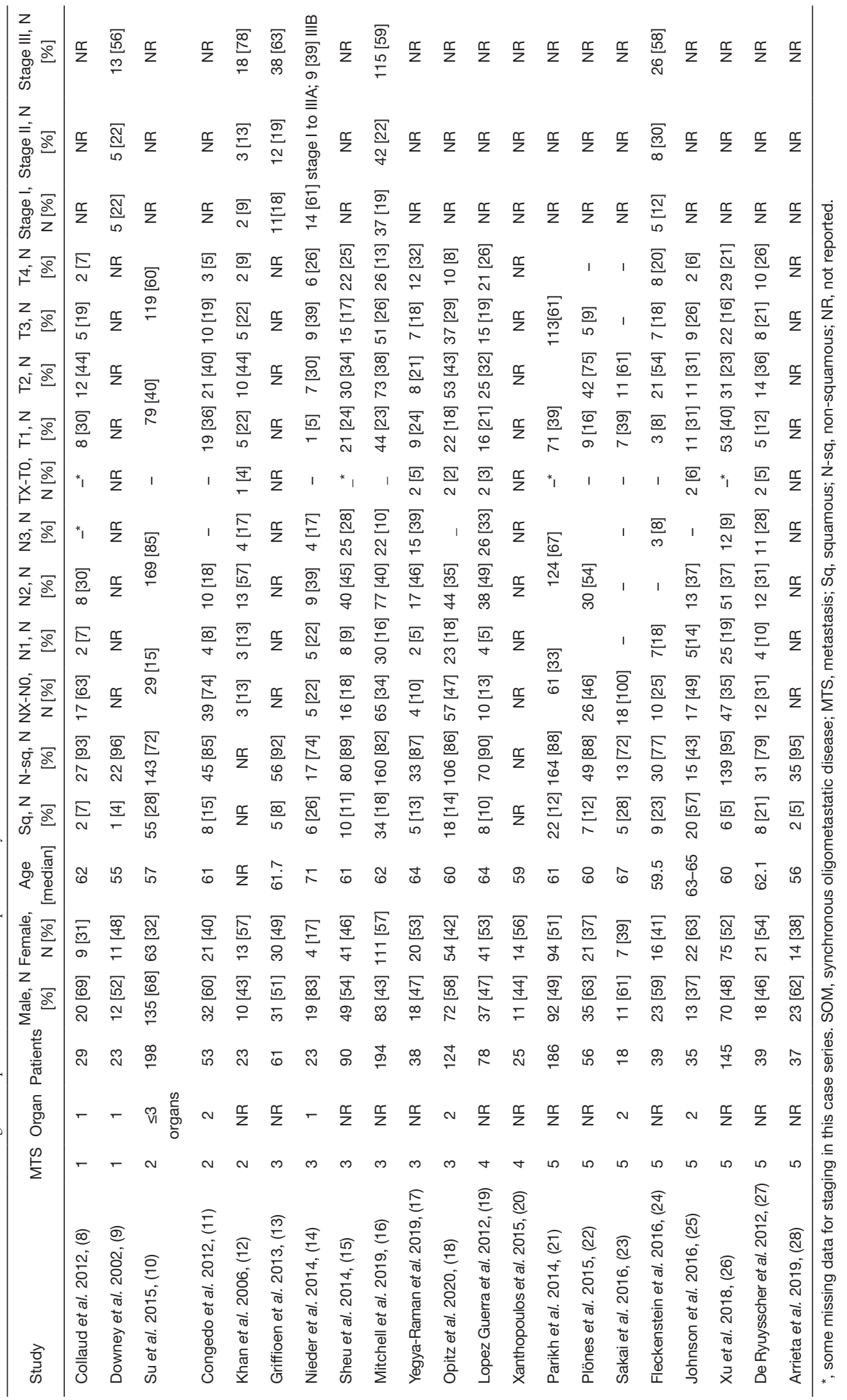


Table 2 Clinical features of OPD lung cancer patients included in in the pooled analysis

\begin{tabular}{|c|c|c|c|c|c|c|c|c|c|c|c|c|}
\hline Authors/year & MTS & Organs & Patients & $\begin{array}{l}\text { Male, } \\
\mathrm{N}[\%]\end{array}$ & $\begin{array}{c}\text { Female, } \\
\text { N [\%] }\end{array}$ & $\begin{array}{c}\text { Age } \\
\text { [median] }\end{array}$ & $\begin{array}{c}\mathrm{Sq}, \\
\mathrm{N}[\%]\end{array}$ & $\begin{array}{l}\mathrm{N}-\mathrm{sq} \\
\mathrm{N}[\%]\end{array}$ & $\begin{array}{c}\text { Stage I, } \\
\text { N [\%] }\end{array}$ & $\begin{array}{c}\text { Stage II, } \\
\text { N [\%] }\end{array}$ & $\begin{array}{c}\text { Stage III, } \\
\text { N [\%] }\end{array}$ & $\begin{array}{c}\text { Stage IV, } \\
\text { N [\%] }\end{array}$ \\
\hline Ni 2019, (29) & 3 & 3 & 71 & $33[46]$ & $38[54]$ & 60 & $3[4]$ & 68 [96] & - & $9[13]$ & $62[87]$ & - \\
\hline Weickhardt 2012, (30) & 4 & NR & 25 & $9[36]$ & $16[64]$ & 58 & NR & NR & NR & NR & NR & NR \\
\hline Yu 2013, (31) & 5 & NR & 18 & $8[44]$ & $10[56]$ & 57 & NR & NR & $2[11]$ & - & $3[17]$ & 13 [72] \\
\hline
\end{tabular}

OPD, oligoprogressive disease; MTS, metastasis; Sq, squamous; N-sq, non-squamous; NR, not reported.

Table 3 Clinical features of ORD lung cancer patients included in in the pooled analysis

\begin{tabular}{lccccccccccc}
\hline Authors/year & MTS & Organs Patients & $\begin{array}{c}\text { Male, } \\
\text { N [\%] }\end{array}$ & $\begin{array}{c}\text { Female, } \\
\text { N [\%] }\end{array}$ & $\begin{array}{c}\text { Age } \\
\text { [median] }\end{array}$ & $\begin{array}{c}\text { Sq, } \\
\text { N [\%] }\end{array}$ & $\begin{array}{c}\text { N-sq, } \\
\text { N [\%] }\end{array}$ & $\begin{array}{c}\text { Stage I, } \\
\text { N [\%] }\end{array}$ & $\begin{array}{c}\text { Stage II, } \\
\text { N [\%] }\end{array}$ & $\begin{array}{c}\text { Stage III, } \\
\text { N [\%] }\end{array}$ \\
\hline Yano et al. 2010, (33) & 1 & 1 & 44 & NR & NR & NR & NR & NR & NR & NR & NR \\
Endo et al. 2014, (34) & 3 & 1 & 13 & $7[54]$ & $6[46]$ & 65 & $4[31]$ & $9[69]$ & NR & NR & NR \\
Shimada et al. 2015, (35) & 5 & 2 & 76 & $46[61]$ & $30[39]$ & 65 & $8[11]$ & $68[89]$ & $36[48]$ & $17[22]$ & $23[30]$ \\
Shang et al. 2018, (36) & 5 & NR & 163 & $128[79]$ & $35[21]$ & NR & $65[40]$ & $98[60]$ & $95[63]$ & - & $56[37]$ \\
\hline
\end{tabular}

ORD, oligorecurrent disease; MTS, metastasis; Sq, squamous; N-sq, non-squamous; NR, not reported.

Table 4 Clinical features of OpeD lung cancer patients included in in the pooled analysis

\begin{tabular}{|c|c|c|c|c|c|c|c|c|c|c|c|c|}
\hline Authors/year & MTS & Organs & Patients & $\begin{array}{l}\text { Male, } \\
\mathrm{N}[\%]\end{array}$ & $\begin{array}{c}\text { Female, } \\
\text { N [\%] }\end{array}$ & $\begin{array}{c}\text { Age } \\
\text { [median] }\end{array}$ & $\begin{array}{c}\text { Sq, } \\
\mathrm{N}[\%]\end{array}$ & $\begin{array}{l}\text { N-sq, } \\
\text { N [\%] }\end{array}$ & $\begin{array}{c}\text { Stage I, } \\
\text { N [\%] }\end{array}$ & $\begin{array}{c}\text { Stage II, } \\
\text { N [\%] }\end{array}$ & $\begin{array}{c}\text { Stage III, } \\
\text { N [\%] }\end{array}$ & MTS \\
\hline Gomez et al. 2019, (37) & 3 & NR & 49 & 22 [45] & 27 [55] & 61 & $5[10]$ & $44[90]$ & NR & NR & NR & NR \\
\hline
\end{tabular}

OpeD, oligo-persistent disease; MTS, metastasis; Sq, squamous; N-sq, non-squamous; NR, not reported.

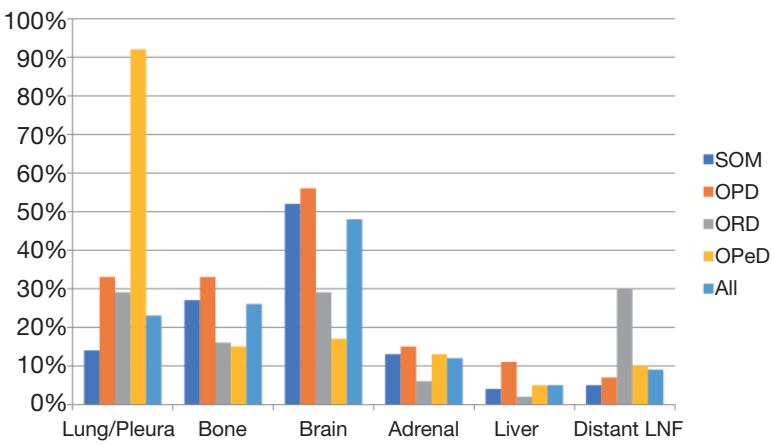

Figure 2 Metastatic organ distribution in oligometastatic patients included in the pooled analysis. Pooled data of oligometastatic NSCLC patients from published case series. Percentages are calculated taking into account only the organs explored in each study. NSCLC, non-small cell lung cancer; LFN, lymph nodes. described in $23 \%$ of patients. Lymph nodes were involved in $9 \%$ of patients, although the selection criteria used may be unclear as, for instance, mediastinal lymph nodes were counted as an organ in only $51 \%$ of articles, and the type of lung/pleural metastatic involvement was not clearly stated in most cases.

Literature data and results of the pooled analyses in each oligometastatic condition are detailed below.

\section{Synchronous oligometastatic disease}

\section{Incidence}

Limited data are available about the incidence of SOM in lung cancer patients. Several case series investigating the efficacy of different locally ablative treatments have not 
provided adequate information about the whole metastatic population. Moreover, the lack of systematic biopsies of the metastatic site and modern imaging methods, such as 18-fluorodeoxyglucose (FDG) positron emission tomography (PET) or brain magnetic resonance, have probably contributed to misestimating SOM incidence.

Hendriks et al. published a population-based analysis of 11,094 NSCLC patients who were metastatic at diagnosis (39). Fifty-one percent $(n=5,676)$ had a single metastatic organ. However, since the number of metastatic lesions was not reported, SOM patients could not be discerned from the overall group.

Conversely, five published case series focused on SOM disease, and reported an incidence ranging from $16 \%$ to $33 \%$ of all metastatic NSCLC at diagnosis $(8,10,14,21,32)$. Unfortunately, any direct comparison would be biased since these studies adopted differing definitions of SOM, ranging from a single metastatic lesion in a single organ up to 5 metastatic lesions or 3 organs involved (Table 1).

Therefore, the main data source available remains the International Association for the Study of Lung Cancer (IASLC) 1999-2010 prospective database (5).

The IASLC cohort included 1,025 non-resected metastatic patients and was able to identify three main subgroups according to the metastatic site and number of metastatic lesions: $32 \%$ of patients presented intra-thoracic metastasis only (M1a), $22 \%$ of patients presented a single extra-thoracic metastasis in a unique organ (M1b), and 46\% presented multiple lesions in a single or multiple organs (22\% and 24\%, respectively) (M1c). Thus, although one can consider patients in the M1b group as SOM according to main consensus definitions and outcome, data are insufficient to effectively isolate SOM patients from the heterogeneous M1a and M1c groups.

\section{Patient clinical features}

In Hendriks et al., single organ metastatic patients were significantly older (mean age 66.1 versus $64.7, \mathrm{P}<0.001$ ), and had both a squamous histology ( $31 \%$ versus $22.1 \%, \mathrm{P}<0.001$ ) and lower $\mathrm{TN}$ status $(18.3 \%$ versus $9.8 \%, \mathrm{P}<0.001)$ more often than patients with two or more organs involved (39).

In our systematic review, 21 articles were selected that focused on SOM and detailed the clinical characteristics of 1,514 patients (Table 1) (8-28). The patients had a median age of 61 years (range, 56-71 years), 54\% ( $\mathrm{N}=814)$ were men and $84 \%(\mathrm{~N}=1,235)$ had a non-squamous histology. With the pooled analysis being mainly composed of SOM diseases, the TN status in this subgroup of patients was closely to the whole cohort. The patients were classified as $\mathrm{N} 0-\mathrm{N} 1$ and $\mathrm{N} 2-\mathrm{N} 3$ in $41 \%(\mathrm{~N}=555)$ and $57 \%(\mathrm{~N}=796)$ of cases respectively, and as T0-T1 in 55\% (N=756) and $\mathrm{T} 2-$ $\mathrm{T} 4(\mathrm{~N}=590)$ in $43 \%$. However, in examining the five articles that reported complete staging of 340 patients, we found more usual proportions with $18 \%$ of patients $(\mathrm{N}=60)$ at stage I, 21\% (N=70) at stage II, and 62\% (N=210) at stage III.

Finally, since surgery is part of the definitive treatment of SOM, we queried the SEER 18-Registry of the US National Cancer Institute (40) to extrapolate data from operated oligometastatic patients (Table 5, refer to Appendix 1 for the strategy used). No specific data were available on the systemic treatments performed. The SEER database outlines a profile for the representative oligometastatic patient, who has a median age of 67 years and is male and Caucasian. In most cases, the patients were diagnosed with adenocarcinoma (pN1) with brain metastasis and received surgical treatments to both the primary tumour and metastatic disease.

However, these epidemiological data should be interpreted with caution given several weaknesses inherent to SEER analyses in general: a lack of information about the performance score and comorbidities of the patients, incomplete data on the distribution of metastases, and the unknown status of concomitant systemic therapy (Table 5). Moreover, surgery is not always a part of definitive SOM treatment (as patients may be medically inoperable or prefer radiotherapy, for example), meaning a profile based on this search would have been biased.

\section{Organs involved in metastatic spread}

Concerning the organs involved in the metastatic spread, the IASLC 1999-2010 cohort provided interesting information on $225 \mathrm{SOM}$ patients presenting a single metastasis in a single extra-thoracic organ (5). In these patients, the most common metastatic organs were the bones $(35.5 \%)$, brain $(25 \%)$, adrenal gland $(20 \%)$ and liver (12\%), whereas in $7.5 \%$ of patients, they were the skin, lymph nodes, or peritoneum (Figure S1). Interestingly, the subset of patients with multiple lesions in a single organ provided a slightly similar snapshot. Bone localization was confirmed as the most common metastatic organ, accounting for $48 \%$ of cases. The brain and liver were involved in about $20 \%$ of patients, whereas the other localizations accounted for less than $20 \%$ of cases globally.

Hendriks et al. reported the metastatic lesion distribution in patients with a single metastatic extra-thoracic organ 
Table 5 Baseline characteristics of patients included in SEER databases

\begin{tabular}{|c|c|}
\hline Patient characteristics & Percentage \\
\hline \multicolumn{2}{|l|}{ Age (years) } \\
\hline$<40$ & 0.73 \\
\hline $40-49$ & 5.37 \\
\hline $50-59$ & 17.99 \\
\hline $60-69$ & 29.64 \\
\hline \multicolumn{2}{|l|}{ Gender } \\
\hline Male & 57.97 \\
\hline Female & 44.03 \\
\hline \multicolumn{2}{|l|}{ Race } \\
\hline White & 79.97 \\
\hline Black & 12.54 \\
\hline Others & 7.32 \\
\hline Unknown & 0.17 \\
\hline \multicolumn{2}{|l|}{ T stage } \\
\hline T0 & 0.70 \\
\hline T1 & 9.07 \\
\hline $\mathrm{T} 2$ & 21.96 \\
\hline T3 & 5.55 \\
\hline $\mathrm{T} 4$ & 48.65 \\
\hline Tx & 14.07 \\
\hline \multicolumn{2}{|l|}{$\mathrm{N}$ stage } \\
\hline No & 23.21 \\
\hline $\mathrm{N} 1$ & 7.59 \\
\hline N2 & 42.42 \\
\hline N3 & 16.79 \\
\hline $\mathrm{Nx}$ & 9.99 \\
\hline \multicolumn{2}{|l|}{ Cause of description as M1 } \\
\hline Distant organ metastases & 84.26 \\
\hline Distant (nonregional) lymph node metastases & 0.70 \\
\hline Pleural/pericardial effusion & 2.10 \\
\hline Contralateral lung nodules & 11.97 \\
\hline Undetermined & 0.97 \\
\hline \multicolumn{2}{|l|}{ Histological subtype } \\
\hline Non-small-cell lung carcinoma, NOS & 22.96 \\
\hline Squamous cell carcinoma variants & 19.76 \\
\hline Adenocarcinoma variants & 54.54 \\
\hline Large cell carcinoma & 2.74 \\
\hline
\end{tabular}

and a single or multiple metastatic site (39). Bones were confirmed to be the most common metastatic sites (28\%) followed by the brain (19\%), whereas the liver, adrenal and distant lymph nodes were involved in less than $10 \%$ (Figure S1).

Our pooled analysis was not limited by the number of metastases or organs involved, and patients may have had metastases in multiple organs. According to our data, the brain was the most common metastatic site (52\%), followed by bones (27\%) (Figure 2 and Figure S1). Less often, patients presented an adrenal localization $(13 \%)$ or liver metastasis (4\%). Distant lymph nodes (5\%) and lung (14\%) involvement were difficult to state, as the selection criteria were heterogeneous and often unclearly defined.

\section{Oligoprogressive and oligorecurrent disease}

Two other oligometastatic presentations can be identified: ORD and OPD. ORD is defined as the appearance of a new and limited metastatic organ after radical treatment or complete remission, while OPD is a metastatic progression during systemic therapy $(41,42)$. The use of local radical treatment in ORD and OPD is usually pursued in daily clinical practice, even though it may delay the prescription of new oncological strategies, and there is insufficient scientific support from evidence-based medicine.

Additionally, there is no uniform and consistent description of the number of recurrent or progressive metastatic lesions and the number of organs involved in ORD and OPD. Consequently, little is known about the incidence and clinical characteristics of these patients, and limited comparison can be done between the prospective clinical studies available. As a matter of fact, most published case series focused on patients presenting one or more metastases in a specific organ. Where multiple metastatic lesions were allowed, the criteria for defining oligometastatic disease often remained undeclared. Further, information regarding the incidence of this condition among the general metastatic population, patient clinical characteristics, and the distribution of metastases is frequently unreported.

\section{Oligoprogressive disease}

Three papers reported the incidence of OPD as ranging from $10 \%$ to $49 \%(30,31,43)$. The first study was a singlearm phase II trial that included 38 locally advanced or metastatic patients treated with oxaliplatin-paclitaxel firstline (43). Seventeen of them (44\%) presented OPD at the 
time of progression, defined as having $\leq 4$ sites of metastatic disease in addition to the primary tumour without pleural/ pericardial effusion.

The second study included metastatic epidermal growth factor receptor (EGFR) and anaplastic lymphoma kinase (ALK)-positive patients $(\mathrm{n}=65)$ who received a firstgeneration tyrosine kinase inhibitor (30). Forty-nine percent of patients that had disease progression at the time of the analysis were classified as OPD according to the following criteria: absence of leptomeningeal involvement, less than four sites of extra-central nervous system (eCNS) progression, good performance status (ECOG $\leq 2$ ) and good therapy tolerance. All these patients underwent a biopsy on the progressive sites and received local ablative treatment (LAT) while continuing the same target agent beyond progression.

The third study (31) included a large cohort $(\mathrm{n}=184)$ of EGFR positive patients progressing during erlotinib or gefitinib. Notably, a small percentage (13\%) presented early-stage disease at diagnosis and there is no information about the treatment setting. This does not exclude the presence of some ORD in this cohort, although most patients had locally advanced or metastatic disease. The authors reported detailed information only about eCNS metastases management, and defined the OPD as having less than 5 metastatic lesions. Ten percent of patients received an LAT for $\mathrm{eCNS}$ OPD, though no information was available about the number of potentially eligible patients, likely underestimating the eCNS OPD incidence. In all these case series, tumour assessment did not include an 18-FDG PET, and only one study requested histological proof of progression.

We also selected 4 case series with exhaustive information on the clinical characteristics of 320 OPD patients (Table 2) (29-32). The series were restricted to oncogene-addicted patients only, mainly EGFR positive. The definition of OPD was different across the studies, and most did not report the incidence. As expected, most patients were women $(54 \%, \mathrm{~N}=173)$ with non-squamous histology (98\%, $\mathrm{N}=272$ ). Staging at initial diagnosis was stage $\mathrm{I}$ in $0.6 \%$ $(\mathrm{N}=2)$, stage II in 3\% (N=9), stage III in $35 \%(\mathrm{~N}=104)$ and stage IV in $61 \%(\mathrm{~N}=180)$ of cases. When assessed, the brain was the most common metastatic site (56\%), followed by bones (33\%) (Figure 1). Adrenal and liver involvement was described in $15 \%$ and $11 \%$ of patients respectively, while distant lymph nodes were metastatic in $7 \%$ of cases. This distribution is consistent with the synchronous oligo-metastatic disease distribution previously described.
Of note, the series reported here used first generation inhibitors; the use of current third generation inhibitors would have possibly changed the metastatic distribution pattern.

\section{Oligorecurrent disease}

Two studies reported the incidence of ORD in surgically resected NSCLC $(35,44)$. Shimada et al. $(35)$ reported 76 cases of ORD among 272 NSCLC patients $(28 \%)$ who relapsed after surgical resection for early disease management. The authors defined ORD as up to 5 metastatic lesions in a maximum of 2 organs that were considered radically curable with the primary disease controlled. However, patients with liver or bone metastases were excluded. This incidence is quite different from the data reported by Suzawa et al. (44), who included only locally-advanced patients that relapsed after a trimodality treatment with up to three metastatic brain lesions or a single eCNS metastasis. Under this definition, ORD represented just $13 \%$ of the relapsing patients (18/137). Concerning the subset of patients with a single metastatic site, two case series reported an impressive relapse incidence of $55 \%$ and $32 \%$, respectively $(45,46)$. The first of these studies was focused on stage I only, while the second included $60 \%$ of stage II/III.

Our pooled has analysis included 4 case series focused on ORD ( $\mathrm{N}=296)$ (Table 3) (33-36). The patients had a median age of 65 years and were mostly men $(72 \%, \mathrm{~N}=181)$ with a non-squamous histology $(69 \%, \mathrm{~N}=175)$. Primary disease was at stage $\mathrm{I}$ in $55 \%(\mathrm{~N}=131)$, II in $7 \%(\mathrm{~N}=17)$ and $\mathrm{III}$ in $34 \%(\mathrm{~N}=79)$ of cases. The most common metastatic sites were distant lymph nodes (30\%), the brain (29\%) and bones $(16 \%)$. Adrenal and liver involvement was present in less than $6 \%$ of cases (Figure 2). Notably, the case series were focused on EGFR mutated NSCLC patients; thus, the data reported are unlikely to be demographically representative of NSCLC patients presenting OPD.

\section{Oligo-persistent disease}

The concept of OPeD is closely related to an oligometastatic state that, after systemic treatment, either persists or is reached from a more widely metastatic state. In fact, many systemically treated NSCLC patients relapse at a pre-existing disease site (47-49). Literature data on OPeD mainly focus on oncogene-addicted NSCLC, assuming that ablating these persistent clones with local therapies could 
improve the overall outcomes (50).

Data on OPeD incidence is not generally available, as clinical trials or studies have focused on patients with a stated oligometastatic disease that subsequently benefited from local treatment on residual sites. The only data available come from the ATOM study, in which EGFR-mutated NSCLC patients received an LAT on the residual lesions after three months of treatment (51). 66 patients in total were screened, and $18(27.3 \%)$ were randomized after three months.

Two studies have been included in the pooled analysis $(\mathrm{N}=78)$ that provide interesting information on the clinical features and organs involved in metastatic spread $(37,38)$. The first is an open-label, randomized phase II trial published by Gomez et al. comparing local consolidative therapy (surgery or radiotherapy) to maintenance therapy or observation. The patients were eligible if they presented three or fewer metastatic disease lesions after first-line systemic treatment. The median age of the population was 61 (range, $48-83$ years), and 27 (55\%) were female. Adenocarcinoma was the most common histology $(\mathrm{n}=39$; $80 \%)$, and $8(16 \%)$ presented an EGFR mutation/ALK translocation. Concerning the number of nonregional metastases after initial systemic therapy, most of the enrolled patients presented 0 or 1 metastatic site $(n=32$; $65 \%)$, and only $27 \%(\mathrm{n}=13)$ had brain metastases (37).

The second study is a phase II randomized clinical trial exploring the combination of consolidative stereotactic radiotherapy and maintenance chemotherapy compared to chemotherapy alone (52). Patients had up to 6 sites of extracranial disease (including primary) with no more than three sites in the liver or lung. Those receiving firstline targeted therapy for EGFR-positive or ALK-positive NSCLC were excluded. A total of 29 patients were enrolled in the study. The median age was 63.5 (range: 51-78) in the combination arm, prevalently male $(n=9 ; 64.3 \%)$ and with a non-squamous histology $(\mathrm{n}=13 ; 92.9 \%)$. The median number of metastatic lesions before induction chemotherapy was 3 (range: $2-6)$, and $57.1 \%(n=8)$ of the patients did not have previously treated brain metastases. A total of 31 lesions were treated in the 14 patients randomized in the combination arm. Intrathoracic sites were the most common locations of treatment of stereotactic radiotherapy.

No information was reported about disease staging in these studies, but the organs involved in the metastatic spread were detailed. Patients had a metastasis to the brain in $17 \%$, bones in $15 \%$, and adrenal gland in $13 \%$ of cases (Table 4). The lymph nodes and liver were less involved at
$10 \%$ and $5 \%$ of patients, respectively.

\section{Discussion}

Oligometastatic status is globally identified as a different clinical condition from multi metastatic NSCLC. It is being progressively recognized that the combination of systemic and local ablative treatments can impact clinical outcomes. Nevertheless, a uniform definition of oligometastic NSCLC is not yet available, thus limiting the correct classification and development of therapeutic strategies. Moreover, the available data are not completely exhaustive, such that to date, it remains challenging to clearly describe the epidemiology of oligometastatic NSCLC.

Few studies have reported incidence information, and the information available is incomparable given the differing oligometastatic definitions employed in each. Considering this limitation, we pooled data from published case series to describe patient clinical features. Globally, patient clinical characteristics are consistent with the general metastatic NSCLC population, but even then, oligometastatic patients seems to have a lower-than-expected TN status. Notably, a different portrait of the organs involved can be obtained based on the specific oligometastatic condition. However, lacking a consensus definition, these results must be interpreted cautiously, and prospective evaluation is urgently needed to drive future clinical trials. An ongoing European study (NCT03818503) is aiming to establish the number of oligometastatic patients eligible for local treatments. A multidisciplinary approach is essential for overcoming current literature limitations and offering the best treatment to this subgroup of patients.

This pooled analysis presents several limitations. While gender, age, histology, and metastatic sites were homogeneously collected in almost all the case series included, few articles clearly reported information about smoking habits, mutations, and performance status. Also, a solid estimation of the true incidence of OMD cannot be determined due to the lack of a denominator.

Furthermore, in a few cases, the authors clearly separated patients with only one metastasis in one organ from those with multiple metastatic lesions in a single or multiple organs. Thus, it was impossible to separately analyze these three conditions in most of the cases. Finally, whereas an exhaustive description of the brain, bone and metastatic adrenal involvement was feasible, information on other distant localizations was less homogeneously collected and, 
in some cases, the selection criteria were unclear.

\section{Acknowledgments}

Funding: None.

\section{Footnote}

Provenance and Peer Review: This article was commissioned by the Guest Editors (Maurizio Infante \& Thierry Berghmans) for the series "Oligometastatic NSCLC: definition and treatment opportunities" published in Translational Lung Cancer Research. The article has undergone external peer review.

Reporting Checklist: The authors have completed the PRISMA reporting checklist. Available at https://dx.doi. org/10.21037/tlcr-20-982

Peer Review File: Available at https://dx.doi.org/10.21037/ tlcr-20-982

Conflicts of Interest: All authors have completed the ICMJE uniform disclosure form (available at https://dx.doi. org/10.21037/tlcr-20-982). The series "Oligometastatic NSCLC: definition and treatment opportunities" was commissioned by the editorial office without any funding or sponsorship. The authors have no other conflicts of interest to declare.

Ethical Statement: The authors are accountable for all aspects of the work in ensuring that questions related to the accuracy or integrity of any part of the work are appropriately investigated and resolved.

Open Access Statement: This is an Open Access article distributed in accordance with the Creative Commons Attribution-NonCommercial-NoDerivs 4.0 International License (CC BY-NC-ND 4.0), which permits the noncommercial replication and distribution of the article with the strict proviso that no changes or edits are made and the original work is properly cited (including links to both the formal publication through the relevant DOI and the license). See: https://creativecommons.org/licenses/by-nc-nd/4.0/.

\section{References}

1. Dingemans AC, Hendriks LEL, Berghmans T, et al.
Definition of Synchronous Oligometastatic Non-Small Cell Lung Cancer-A Consensus Report. J Thorac Oncol 2019;14:2109-19.

2. Guckenberger $M$, Lievens $Y$, Bouma AB, et al. Characterisation and classification of oligometastatic disease: a European Society for Radiotherapy and Oncology and European Organisation for Research and Treatment of Cancer consensus recommendation. Lancet Oncol 2020;21:e18-28.

3. Tree AC, Khoo VS, Eeles RA, et al. Stereotactic body radiotherapy for oligometastases. Lancet Oncol 2013;14:e28-37.

4. Ashworth AB, Senan S, Palma DA, et al. An individual patient data metaanalysis of outcomes and prognostic factors after treatment of oligometastatic non-small-cell lung cancer. Clin Lung Cancer 2014;15:346-55.

5. Eberhardt WEE, Mitchell A, Crowley J, et al. The IASLC Lung Cancer Staging Project: Proposals for the Revision of the M Descriptors in the Forthcoming Eighth Edition of the TNM Classification of Lung Cancer. J Thorac Oncol 2015;10:1515-22.

6. deSouza NM, Liu Y, Chiti A, et al. Strategies and technical challenges for imaging oligometastatic disease: Recommendations from the European Organisation for Research and Treatment of Cancer imaging group. Eur J Cancer 2018;91:153-63.

7. Ashworth A, Rodrigues G, Boldt G, et al. Is there an oligometastatic state in non-small cell lung cancer? A systematic review of the literature. Lung Cancer 2013;82:197-203.

8. Collaud S, Stahel R, Inci I, et al. Survival of patients treated surgically for synchronous single-organ metastatic NSCLC and advanced pathologic TN stage. Lung Cancer 2012;78:234-8.

9. Downey RJ, Ng KK, Kris MG, et al. A phase II trial of chemotherapy and surgery for non-small cell lung cancer patients with a synchronous solitary metastasis. Lung Cancer 2002;38:193-7.

10. Su S, Li T, Lu B, et al. Three-Dimensional Radiation Therapy to the Primary Tumor With Concurrent Chemotherapy in Patients With Stage IV Non-Small Cell Lung Cancer: Results of a Multicenter Phase 2 Study From PPRA-RTOG, China. Int J Radiat Oncol Biol Phys 2015;93:769-77.

11. Congedo MT, Cesario A, Lococo F, et al. Surgery for oligometastatic non-small cell lung cancer: longterm results from a single center experience. J Thorac Cardiovasc Surg 2012;144:444-52. 
12. Khan AJ, Mehta PS, Zusag TW, et al. Long term disease-free survival resulting from combined modality management of patients presenting with oligometastatic, non-small cell lung carcinoma (NSCLC). Radiother Oncol 2006;81:163-7.

13. Griffioen GH, Toguri D, Dahele M, et al. Radical treatment of synchronous oligometastatic non-small cell lung carcinoma (NSCLC): patient outcomes and prognostic factors. Lung Cancer 2013;82:95-102.

14. Nieder C, Tollåli T, Reigstad A, et al. Oligometastatic non-small cell lung cancer: a significant entity outside of specialized cancer centers? Med Princ Pract 2014;23:526-31.

15. Sheu T, Heymach JV, Swisher SG, et al. Propensity scorematched analysis of comprehensive local therapy for oligometastatic non-small cell lung cancer that did not progress after front-line chemotherapy. Int J Radiat Oncol Biol Phys 2014;90:850-7.

16. Mitchell KG, Farooqi A, Ludmir EB, et al. Improved Overall Survival With Comprehensive Local Consolidative Therapy in Synchronous Oligometastatic Non-Small-Cell Lung Cancer. Clin Lung Cancer 2020;21:37-46.e7.

17. Yegya-Raman N, Aisner J, Kim S, et al. Synchronous Oligometastatic Non-small Cell Lung Cancer Managed With Curative-Intent Chemoradiation Therapy: Longterm Outcomes From a Single Institution. Adv Radiat Oncol 2019;4:541-50.

18. Opitz I, Patella M, Payrard L, et al. Prognostic factors of oligometastatic non-small-cell lung cancer following radical therapy: a multicentre analysis. Eur J Cardiothorac Surg 2020;57:1166-72.

19. Lopez Guerra JL, Gomez D, Zhuang Y, et al. Prognostic impact of radiation therapy to the primary tumor in patients with non-small cell lung cancer and oligometastasis at diagnosis. Int J Radiat Oncol Biol Phys 2012;84:e61-7.

20. Xanthopoulos EP, Handorf E, Simone CB 2nd, et al. Definitive dose thoracic radiation therapy in oligometastatic non-small cell lung cancer: A hypothesisgenerating study. Pract Radiat Oncol 2015;5:e355-63.

21. Parikh RB, Cronin AM, Kozono DE, et al. Definitive primary therapy in patients presenting with oligometastatic non-small cell lung cancer. Int J Radiat Oncol Biol Phys 2014;89:880-7.

22. Plönes T, Osei-Agyemang T, Krohn A, et al. Surgical Treatment of Extrapulmonary Oligometastatic Non-small Cell Lung Cancer. Indian J Surg 2015;77:216-20.

23. Sakai K, Takeda M, Hayashi H, et al. Clinical outcome of node-negative oligometastatic non-small cell lung cancer.
Thorac Cancer 2016;7:670-5.

24. Fleckenstein J, Petroff A, Schäfers HJ, et al. Longterm outcomes in radically treated synchronous vs. metachronous oligometastatic non-small-cell lung cancer. BMC Cancer 2016 02;16:348.

25. Johnson KK, Rosen JE, Salazar MC, et al. Outcomes of a Highly Selective Surgical Approach to Oligometastatic Lung Cancer. Ann Thorac Surg 2016;102:1166-71.

26. Xu Q, Zhou F, Liu H, et al. Consolidative Local Ablative Therapy Improves the Survival of Patients With Synchronous Oligometastatic NSCLC Harboring EGFR Activating Mutation Treated With First-Line EGFRTKIs. J Thorac Oncol 2018;13:1383-92.

27. De Ruysscher D, Wanders R, van Baardwijk A, et al. Radical treatment of non-small-cell lung cancer patients with synchronous oligometastases: long-term results of a prospective phase II trial (Nct01282450). J Thorac Oncol 2012;7:1547-55.

28. Arrieta O, Barrón F, Maldonado F, et al. Radical consolidative treatment provides a clinical benefit and long-term survival in patients with synchronous oligometastatic non-small cell lung cancer: A phase II study. Lung Cancer 2019;130:67-75.

29. Ni Y, Liu B, Ye X, et al. Local Thermal Ablation with Continuous EGFR Tyrosine Kinase Inhibitors for EGFRMutant Non-small Cell Lung Cancers that Developed Extra-Central Nervous System (CNS) Oligoprogressive Disease. Cardiovasc Intervent Radiol 2019;42:693-9.

30. Weickhardt AJ, Scheier B, Burke JM, et al. Local ablative therapy of oligoprogressive disease prolongs disease control by tyrosine kinase inhibitors in oncogene-addicted non-smallcell lung cancer. J Thorac Oncol 2012;7:1807-14.

31. Yu HA, Sima CS, Huang J, et al. Local therapy with continued EGFR tyrosine kinase inhibitor therapy as a treatment strategy in EGFR-mutant advanced lung cancers that have developed acquired resistance to EGFR tyrosine kinase inhibitors. J Thorac Oncol 2013;8:346-51.

32. Xu Q, Liu H, Meng S, et al. First-line continual EGFR-TKI plus local ablative therapy demonstrated survival benefit in EGFR-mutant NSCLC patients with oligoprogressive disease. J Cancer 2019;10:522-9.

33. Yano T, Haro A, Yoshida T, et al. Prognostic impact of local treatment against postoperative oligometastases in non-small cell lung cancer. J Surg Oncol 2010;102:852-5.

34. Endo C, Hasumi T, Matsumura Y, et al. A prospective study of surgical procedures for patients with oligometastatic non-small cell lung cancer. Ann Thorac Surg 2014;98:258-64. 
35. Shimada Y, Saji H, Kakihana M, et al. Survival outcomes for oligometastasis in resected non-small cell lung cancer. Asian Cardiovasc Thorac Ann 2015;23:937-44.

36. Shang S, Su Y, Zhu Z, et al. Local ablative therapy with or without chemotherapy for non-small-cell lung cancer patients with postoperative oligometastases. Cancer Manag Res 2018;10:6421-9.

37. Gomez DR, Tang C, Zhang J, et al. Local Consolidative Therapy Vs. Maintenance Therapy or Observation for Patients With Oligometastatic Non-Small-Cell Lung Cancer: Long-Term Results of a Multi-Institutional, Phase II, Randomized Study. J Clin Oncol 2019;37:1558-65.

38. Iyengar P, Kavanagh BD, Wardak Z, et al. Phase II trial of stereotactic body radiation therapy combined with erlotinib for patients with limited but progressive metastatic non-small-cell lung cancer. J Clin Oncol 2014;32:3824-30.

39. Hendriks LE, Derks JL, Postmus PE, et al. Single organ metastatic disease and local disease status, prognostic factors for overall survival in stage IV non-small cell lung cancer: Results from a population-based study. Eur J Cancer 2015;51:2534-44.

40. SEER Cancer Statistics Review, 1975-2017 [Internet]. SEER. [cited 2020 May 20]. Available online: https://seer. cancer.gov/csr/1975_2017/index.html

41. Niibe Y, Hayakawa K. Oligometastases and oligorecurrence: the new era of cancer therapy. Jpn J Clin Oncol 2010;40:107-11.

42. Ricardi U, Giaj Levra N, Badellino S, et al. Role of consolidative stereotactic ablative radiotherapy in patients with oligometastatic non-small cell lung cancer. J Thorac Dis 2017;9:2235-7.

43. Mehta N, Mauer AM, Hellman S, et al. Analysis of further disease progression in metastatic non-small cell lung cancer: implications for locoregional treatment. Int J Oncol 2004;25:1677-83.

Cite this article as: Gobbini E, Bertolaccini L, Giaj-Levra N, Menis J, Giaj-Levra M. Epidemiology of oligometastatic nonsmall cell lung cancer: results from a systematic review and pooled analysis. Transl Lung Cancer Res 2021;10(7):3339-3350. doi: $10.21037 /$ tlcr-20-982
44. Suzawa K, Soh J, Takahashi Y, et al. Clinical outcome of patients with recurrent non-small cell lung cancer after trimodality therapy. Surg Today 2019;49:601-9.

45. Nakagawa T, Okumura N, Ohata K, et al. Postrecurrence survival in patients with stage I non-small cell lung cancer. Eur J Cardiothorac Surg 2008;34:499-504.

46. Yano T, Okamoto T, Haro A, et al. Local treatment of oligometastatic recurrence in patients with resected nonsmall cell lung cancer. Lung Cancer 2013;82:431-5.

47. Al-Halabi H, Sayegh K, Digamurthy SR, et al. Pattern of Failure Analysis in Metastatic EGFR-Mutant Lung Cancer Treated with Tyrosine Kinase Inhibitors to Identify Candidates for Consolidation Stereotactic Body Radiation Therapy. J Thorac Oncol 2015;10:1601-7.

48. Patel SH, Rimner A, Foster A, et al. Patterns of initial and intracranial failure in metastatic EGFR-mutant nonsmall cell lung cancer treated with erlotinib. Lung Cancer 2017;108:109-14.

49. Rusthoven KE, Hammerman SF, Kavanagh BD, et al. Is there a role for consolidative stereotactic body radiation therapy following first-line systemic therapy for metastatic lung cancer? A patterns-of-failure analysis. Acta Oncol 2009; 48:578-83.

50. Campo M, Al-Halabi H, Khandekar M, et al. Integration of Stereotactic Body Radiation Therapy With Tyrosine Kinase Inhibitors in Stage IV Oncogene-Driven Lung Cancer. Oncologist 2016;21:964-73.

51. Chan OSH, Lam KC, Li JYC, et al. ATOM: A phase II study to assess efficacy of preemptive local ablative therapy to residual oligometastases of NSCLC after EGFR TKI. Lung Cancer 2020;142:41-6.

52. Iyengar P, Wardak Z, Gerber DE, et al. Consolidative Radiotherapy for Limited Metastatic Non-Small-Cell Lung Cancer: A Phase 2 Randomized Clinical Trial. JAMA Oncol 2018;4:e173501. 


\section{Appendix 1: Sorting strategy for the SEER database query}

SEER ${ }^{*}$ Stat software (version 8.3.6.1) was used to select the cases from the database with the methodology reported elsewhere (1). Cases with any of the histological subtypes of NSCLC including only patients with unspecified NSCLC, adenocarcinoma, squamous cell carcinoma, large cell carcinoma, or any of their variants were retrieved using the variable ICD-0-3 Hist/ Behav, malignant. The search was then limited to cases with the lung and bronchus as primary sites, using the variable site and morphology, site recode, ICD-O-3/WHO 2008, stage IV patient at presentation, any $\mathrm{T}$ and any $\mathrm{N}$, and was restricted to 1995-2016. Types of surgical treatments to the primary included lobectomy and pneumonectomy. Data about definitive radiotherapy to the primary could not be extracted from the database.

The SEER database classifies surgical treatments for metastatic disease into either surgical treatment on nonregional lymph nodes or other distant sites (including contralateral lung). The type of local therapy to the primary was recognized using "surgery of the primary site" as the variable, while the type of surgical treatment for the metastatic disease was identified using the variable "Rx Summary other Regional/distant disease". Cause of designation as M1 disease was identified according to the variable "CS mets at DX". Additional data about sites of distant organ involvement were available for cases diagnosed after 2010 (only for liver, brain, and bone metastases). No specific data were available about suprarenal deposits. Data about systemic therapies were not accessible in the SEER database. Patients' stage information was based on the AJCC stage grouping as recorded in the SEER database.

\section{References}

1. Abdel-Rahman O. Outcomes of Surgery as Part of the Management of Metastatic Non-Small-Cell Lung Cancer: A Surveillance, Epidemiology and End Results Database Analysis. Cancer Invest. 2018;36:238-45.

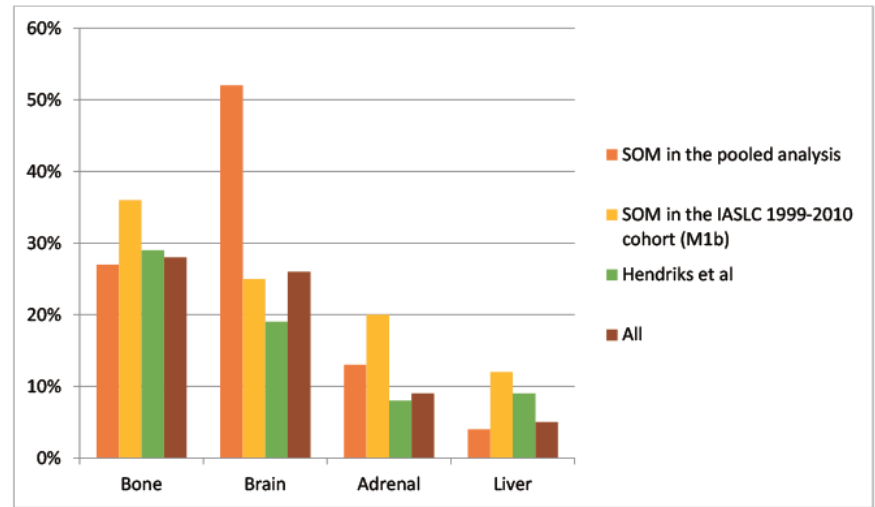

Figure S1 Metastatic organ distribution in oligometastatic patients included in the pooled analysis and in the IASLC 1999-2010 cohort (M1b group). Percentages are calculated taking into account only the organs explored in each study. 